\title{
Development of a Novel Immunochromatographic Assay for Rapid Detection of OXA-23 $\beta$-lactamase-producing Acinetobacter baumannii
}

\author{
Gil Young $\mathrm{Ji}^{1}$, Hyung Geun Song ${ }^{1}$, Mi Young $\mathrm{Jo}^{2}$, Seung Bok Hong ${ }^{3}$ and Kyeong Seob Shin ${ }^{4, \dagger}$ \\ ${ }^{I}$ Department of Pathology, Chungbuk National University College of Medicine, Cheongju 28644, Korea \\ ${ }^{2}$ Department of Physiology, Chungbuk National University College of Medicine, Cheongju 28644, Korea \\ ${ }^{3}$ Department of Clinical Laboratory Science, Chungbuk Health \& Science University, Cheongju 28150, Korea \\ ${ }^{4}$ Department of Laboratory Medicine, Chungbuk National University College of Medicine, Cheongju 28644, Korea
}

Among the several agents causing carbapenem resistance of Acinetobacter baumannii, the most common cause is OXA-23 $\beta$-lactamase, which is known to hydrolyze carbapenem. To effectively control dissemination of carbapenemresistant Acinetobacter baumannii (CRAB), development of both rapid and easy-to-use detection methods are required. The aim of this study is to develop a novel immunochromatographic assay (ICA) for rapid detection of OXA-23 $\beta$-lactamase. Of the seven monoclonal antibodies (mAbs) screened by ELISA, four mAbs (4G6, 4H6, 6G4, 9A4) exhibited high reactivity. Of these four specific antibodies, the combination of 6G4/4G6 showed the greatest reactivity and this combination of mAbs (6G4/4G6 mAbs) was used to develop the OXA-23 $\beta$-lactamase ICA. Of 102 A. baumannii isolates tested, the OXA-23 $\beta$-lactamase ICA results were consistent with PCR analysis except one false positive and one false negative isolate. The overall sensitivity and specificity were $98.36 \%$ and $97.56 \%$, respectively. In conclusion, to the best of our knowledge, we have developed the first specific antibody set to detect OXA-23 $\beta$-lactamase using an ICA kit. This novel ICA can be used as a reliable and easy-to-use immunological assay for detection of OXA-23 $\beta$-lactamase producing CRAB in clinical laboratories.

Key Words: Acinetobacter baumannii, OXA-23 $\beta$-lactamase, Immunochromatographic assay, Rapid detection

\section{INTRODUCTION}

Acinetobacter baumannii frequently causes various nosocomial infections worldwide, including ventilator-associated pneumonia, bacteremia, and urinary tract infections (MunozPrice and Weinstein, 2008; Peleg et al., 2008; Villegas and Hartstein, 2003). In particular, nosocomial infections caused by this species often occur in patients hospitalized in intensive care and burn units (Wilks et al., 2006). Recently, the prevalence of multidrug-resistance in A. baumannii has dramatically increased (Lee et al., 2006; Lee et al., 2009; Lee et al., 2009; Sung et al., 2011). Carbapenem-resistant Acinetobacter spp. are increasing due to the emergence of carbapenem-hydrolyzing $\beta$-lactamases belonging to molecular classes B and D (Walther-Rasmussen and Hoiby, 2006; Lee et al., 2009). Whereas class B carbapenemase, a metallo- $\beta$-lactamase (MBL), have been frequently founded in non-baumannii members of this genus, an increase in class D carbapenemases has been reported in A. baumannii in South Korea (Lee et al., 2009; Lee et al., 2007). It is known that class D carbapenemase in A. baumannii and

\footnotetext{
* Received: January 9, 2016 / Revised: February 27, 2016 / Accepted: February 29, 2016

${ }^{\dagger}$ Corresponding author: Kyeong Seob Shin. Department of Laboratory Medicine, Chungbuk National University College of Medicine, Cheongju 28644 , Korea. Tel: +82-43-269-6240, Fax: +82-43-271-5243, e-mail: ksshin@chungbuk.ac.kr

(C) The Korean Society for Biomedical Laboratory Sciences. All rights reserved.

(c) This is an Open Access article distributed under the terms of the Creative Commons Attribution Non-Commercial License (http://creativecommons.org/licenses/by-nc/3.0/) which permits unrestricted non-commercial use, distribution, and reproduction in any medium, provided the original work is properly cited.
} 
Table 1. Oligonucleotide primers used in this study to detect OXA type carbapenemase

\begin{tabular}{|c|c|c|c|}
\hline Name & Primer sequence $\left(5^{\prime} \rightarrow 3^{\prime}\right)$ & Target gene & Reference \\
\hline OXA23-F & GAT CGG ATT GGA GAA CCA GA & \multirow[b]{2}{*}{$b l a_{\text {OXA-23-like }}$} & \multirow{2}{*}{ (Woodford et al., 2006) } \\
\hline OXA23-R & ATT TCT GAC CGC ATT TCC AT & & \\
\hline OXA24-F & GGT TAG TTG GCC CCC TTAAA & \multirow{2}{*}{$b l a_{\text {OXA-24 }}$-like } & \multirow{2}{*}{ (Woodford et al., 2006) } \\
\hline OXA24-R & AGT TGA GCG AAA AGG GGA TT & & \\
\hline OXA51-F & TAA TGC TTT GAT CGG CCT TG & \multirow{2}{*}{$b l a_{\mathrm{OXA}-51}-$ like } & \multirow{2}{*}{ (Woodford et al., 2006) } \\
\hline OXA51-R & TGG ATT GCA CTT CAT CTT GG & & \\
\hline OXA58-F & AAG TAT TGG GGC TTG TGC TG & \multirow{2}{*}{$b l a_{\text {OXA-58 }}-$ like } & \multirow{2}{*}{ (Woodford et al., 2006) } \\
\hline OXA58-R & CCC CTC TGC GCT CTA CAT AC & & \\
\hline
\end{tabular}

class B carbapenemase in non-baumannii are principal agents which cause the resistance to carbapenem in these strains (Lee et al., 2009). The OXA-type class D carbapenemases of $A$. baumannii can be divided into the following subgroups: OXA-23, OXA-24, OXA-51 and OXA-58. Of these, OXA-23 and OXA-51 are the most common and frequently occur simultaneously within a single isolate. While OXA-51 is an intrinsic enzyme, OXA-23 is an acquired enzyme and it frequently leads to $A$. baumannii outbreaks in intensive care units around the world (Jeong et al., 2006; Lee et al., 2009; Zong et al., 2008).

In this study, we designed an immunochromatographic assay using novel monoclonal antibodies (mAbs) for OXA$23 \beta$-lactamase and evaluated the performance of the rapid kit using carbapenem-resistant Acinetobacter baumannii.

\section{MATERIALS AND METHODS}

\section{Bacterial isolates}

A total of 102 non-duplicated A. baumannii strains resistant to carbapenem (including imipenem or meropenem) were collected from hospital in South Korea. The identification of all strains was initially carried out by biochemical tests using the Vitek II system (bioMérieux, Hazelwood, MO, USA), and subsequently confirmed by the detection of $b l a_{\text {OXA-51 }}$ gene (Turton et al., 2006). The susceptibility of the strains to carbapenem or the other antibiotics was screened by disk diffusion method or Vitek II susceptibility card (CLSI, 2012). The confirmative test of antibiotics including imipenem was performed by E-test (bioMérieux, Inc., Durharm,
NC, USA).

Antimicrobial susceptibility testing and screening for carbapenemase production

The minimal inhibitory concentrations (MICs) of imipenem were determined for the bacterial isolates by the Clinical and Laboratory Standards Institute (CLSI) E-test or broth dilution method (CLSI, 2012). Escherichia coli ATCC 25922 and Pseudomonas aeruginosa ATCC 27853 were used as reference strains for antimicrobial susceptibility testing. The modified IPM disk Hodge test was used to screen for carbapenemase and IPM-EDTA double disk synergy test was used to screen for MBL (Lee et al., 2003).

\section{Amplification and sequencing of antimicrobial resistance genes}

The genes encoding OXA-type carbapenemases were detected through a multiplex PCR using the genomic DNAs as templates and the reaction conditions were described by Woodford et al. (Woodford et al., 2006). GeneAmp 9700 PCR system (Applied Biosystems, Foster City, CA, USA) was used for the amplification and the primers and PCR reaction conditions were described in Table 1. The amplified DNA products were purified according to the manufacturer's instruction (Promega, Madison, WI, USA) and sequenced using an ABI3130XL automated DNA sequencer (Applied Biosystems, Foster City, CA, USA). The sequences were compared with those in the GenBank nucleotide database (www.ncbi.nlm.nih.gov/blast/). In addition, previously reported PCR primers were used to determine the presence 
of $b l a_{\mathrm{IMP}-1}, b l a_{\mathrm{VIM}-1}$, or $b l a_{\mathrm{VIM}-2}$ (Yum et al., 2002), which is class B carbapenemase (metallo- $\beta$-lactamase).

Expression and purification of recombinant OXA-23

\section{$\beta$-lactamase}

The genes encoding the OXA-23 $\beta$-lactamase originated from A. baumannii was synthesized (BioBasic Inc., Toronto, Canada). A synthesized gene of $822 \mathrm{bp}$, corresponding to OXA-23 $\beta$-lactamase, was directly ligated into the pET101/ D-TOPO cloning and expression vector (Invitrogen, CA, USA) by the heat-shock method (Bergmans et al., 1981). The ligation products were transformed into competent Escherichia coli BL21 Star ${ }^{\mathrm{TM}}$ (DE3) cells (Invitrogen), and the transformants were selected on LB agar containing 50 $\mu \mathrm{g} / \mathrm{mL}$ ampicillin. Expressed histidine-tagged proteins were purified by immobilized metal ion affinity chromatography (Hochuli et al., 1988). The purified fraction from Ni-resin affinity chromatography was confirmed by $12 \%$ SDS PAGE, and a band was obtained corresponding to about $30 \mathrm{kDa}$.

\section{Preparation of monoclonal or polyclonal antibodies specific for OXA-23 $\beta$-lactamase}

Anti-OXA-23 mAbs were prepared as described previously (Stähli et al., 1980). Purified His-tagged recombinant OXA$23 \beta$-lactamase was used for immunization and screening of hybridomas by indirect enzyme-linked immunosorbent

(A)

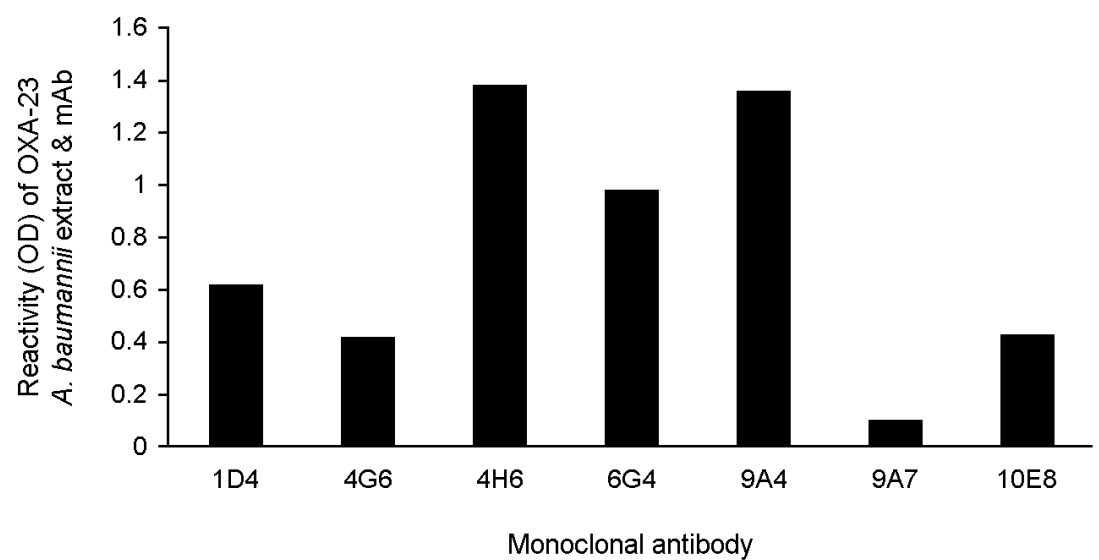

B

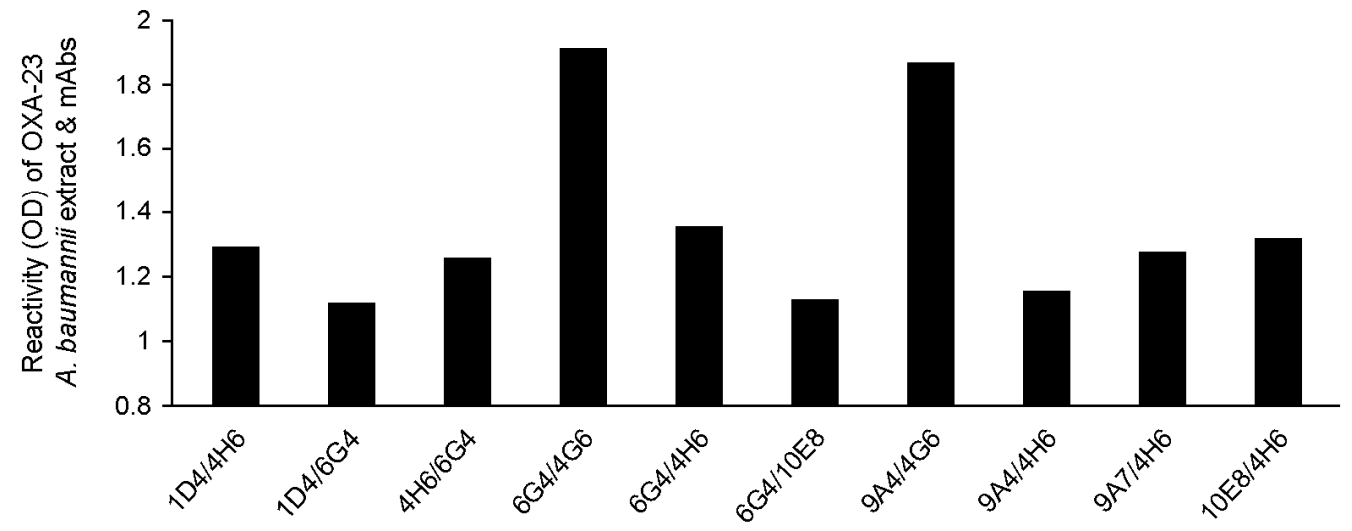

Pair of monoclonal antibody

Fig. 1. Selection of monoclonal antibody and pair to detect OXA-23 $\beta$-lactamase by indirect and sandwich ELISA. Seven mAbs selected by ELISA showed reactivity (OD) of OXA-23 producing $A$. baumannii extract and mAbs (A). A pair of mA 6G4/4G6 revealed the greatest reactivity $(\mathrm{B})$. 


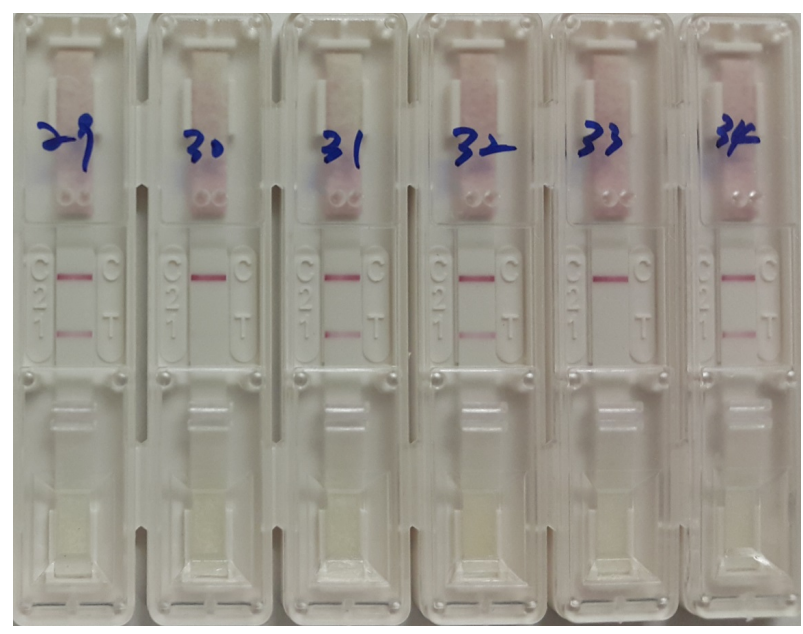

Fig. 2. The results of immunochromatographic assay to detect OXA-23 $\beta$-lactamase in carbapenem-resistant Acinetobacter baumannii. Six $A$. baumannii isolates were tested using the developed ICA kit. Isolate \#29, \#31, \#32, and \#34 (bla $a_{\mathrm{OXA}-23}$ gene positive) show two distinct bands on the control and test line (positive result) but isolate \#30 and isolate \#33 isolates (bla OXA-23 $_{\text {nega- }}$ tive) only show a band in control line (negative result).

assay (ELISA). Among the anti-OXA-23 mAbs selected by ELISA, various $\mathrm{mAbs}$ combinations were evaluated for detection of OXA-23 $\beta$-lactamase. The animal experiments were approved by the Ethical Committee for Animal Experiments at the Research Institute of Dinona Co. (approval number: DN13-RD-CH05).

\section{Determination of mAbs to detect OXA-23 $\beta$-lactamase and preparation of the ICA kit}

The best combination of mAbs acting as antibody for OXA-23 $\beta$-lactamase was determined by indirect ELISA and sandwich ELISA. To improve the sensitivity of ICA, rabbit polyclonal antibody was induced by recombinant OXA-23 $\beta$-lactamase and then this polyclonal antibody was used as the capture antibody. To prepare the test and control lines of ICA, $1.0 \mathrm{mg}$ of rabbit poly-antibody specific for OXA-23 $\beta$-lactamase (as the capture antibody) and $0.3 \mathrm{mg}$ of anti-mouse IgG (Dinona, Iksan, South Korea) per test were coated onto a nitrocellulose membrane (Millipore, Billerica, MA, USA) at $30 \mathrm{~mm}$ and $35 \mathrm{~mm}$ distal from the sample application area, respectively. Gold colloid conjugated with anti-OXA-23 $\beta$-lactamase monoclonal $\operatorname{IgG}$ (as the detector antibody) was sprayed onto a conjugated pad (Millipore) placed on an adjacent sample pad and lyophilized overnight.

\section{Assessement of the immunochromatographic assay using clinical isolates}

To evaluate the performance of ICA for OXA-23 $\beta$ lactamase, the sensitivity and specificity were determined using carbapenem resistant $A$. baumannii with PCR for $b l a_{\mathrm{OXA}-23}$ in two laboratories. To evaluate the cross reactivity with other bacteria, the carbapenem resistant Pseudomonas species, Achromobacter xylosoxidans and the other gram negative or positive bacteria were tested by ICA for OXA-23 (Table 3).

\section{RESULTS}

\section{Selection of anti-OXA-23 $\beta$-lactamase mAbs and pre- paration of ICA kit}

The seven mAbs for OXA-23 $\beta$-lactamase were selected by indirect ELISA. To determine the specific antibody combination for OXA-23 $\beta$-lactamase, various $\mathrm{mAb}$ pairs from the seven mAbs were evaluated with the extract of OXA-23 A. baumannii. Four antibodies such as 4G6, 4H6, 6G4, 9A4 revealed great reactivity for OXA-23 $\beta$-lactamase. Among four mAbs (4G6, 4H6, 6G4, 9A4), 6G4/4G6 pair showed the greatest reactivity (Fig. 1). In addition to, a rabbit antiOXA-23 polyclonal antibody was induced by recombinant OXA-23 $\beta$-lactamase and then used for capture antibody to improve the sensitivity of ICA. To develop ICA kit, rabbit anti-OXA-23 polyclonal antibody was immobilized on the nitrocellulose membrane for capture and four mAbs (4G6, 4H6, 6G4, 9A4) were labeled with colloidal-gold particle as the detector of OXA-23. Of the four combinations, rabbit polyclonal antibody capture plus 6G4 detector showed the greatest reactivity against the extract of OXA-23 producing A. baumannii; therefore, this combination was used to prepare the OXA-23 $\beta$-lactamase ICA (Fig. 2).

\section{Assessement of the assay using clinical isolates}

Of 102 A. baumannii, 61 were positive for $b l a_{\mathrm{OXA}-23}$ gene and 41 isolates were negative for $b a_{\mathrm{OXA}-23}$ gene by PCR 
Table 2. Diagnostic performance of the immunochromatographic assay for OXA-23 $\beta$-lactamase in clinical isolates cultured from MacConkey agar compared with PCR detection of OXA-23 $\beta$-lactamase producing Acinetobacter baumannii

\begin{tabular}{|c|c|c|c|c|c|}
\hline \multirow{3}{*}{ Microorganisms (n) } & \multirow{3}{*}{ PCR for $b l a_{\mathrm{OXA}-23}$} & \multicolumn{4}{|c|}{ ICA for OXA-23 $\beta$-lactamase } \\
\hline & & \multicolumn{2}{|c|}{ A-Lab } & \multicolumn{2}{|c|}{ B-Lab } \\
\hline & & $\mathrm{P}$ & $\mathrm{N}$ & $\mathrm{P}$ & $\mathrm{N}$ \\
\hline \multirow{4}{*}{ CR-Acinetobacter baumannii (102) } & $\mathrm{P}$ & 60 & 1 & 59 & 2 \\
\hline & $\mathrm{N}^{*}$ & 1 & 40 & 0 & 41 \\
\hline & Sensitivity (\%) & \multicolumn{2}{|c|}{98.36} & \multicolumn{2}{|c|}{96.72} \\
\hline & Specificity (\%) & \multicolumn{2}{|c|}{97.56} & \multicolumn{2}{|c|}{100} \\
\hline CR-Pseudomonas spp. (33) $)^{\dagger}$ & $\mathrm{N}$ & 0 & 33 & 0 & 33 \\
\hline CR-Achromobacter xylosoxidans (9) & $\mathrm{N}$ & 0 & 9 & 0 & 9 \\
\hline \multirow{4}{*}{ Total (144) } & $\mathrm{P}$ & 60 & 1 & 59 & 2 \\
\hline & $\mathrm{N}$ & 1 & 82 & 0 & 83 \\
\hline & Sensitivity (\%) & \multicolumn{2}{|c|}{98.36} & \multicolumn{2}{|c|}{96.72} \\
\hline & Specificity (\%) & \multicolumn{2}{|c|}{98.80} & \multicolumn{2}{|c|}{100} \\
\hline
\end{tabular}

Abbreviations: CR, carbapenem resistant; ICA, immunochromatographic assay; P, positive; N, negative

*Among 41 CR-A. baumannii without bla ${ }_{\text {OXA-23 }}$ included 19 IMP-1 and 13 VIM-2 type metallo- $\beta$-lactamase (MBL) producing isolates.

${ }^{\dagger}$ CR-Pseudomonas (P. aeruginosa, P. fluorescens and P. putida) spp. included 32 VIM-2 type MBL producing isolates.

${ }^{\ddagger}$ All of CR-A. xylosoxidans was VIM-2 type MBL producing isolates.

analysis. Sixty isolates among 61 isolates with $b l a_{\mathrm{OXA}-23}$ gene were positive by ICA. Forty isolates among 41 isolates without bla $_{\mathrm{OXA}-23}$ gene were negative by ICA in A-laboratory. In B-laboratory, 100/102 was consistent with PCR results except 2 false negative. The overall sensitivity and specificity were $98.36 \%$ and $97.56 \%$ in A-laboratory, $96.72 \%$ and $100 \%$ in B-laboratory, respectively (Table 2). To evaluate cross reactivity with other bacteria, the CR-P. aeruginosa, A. xylosoxidans and the other gram negative or positive bacteria were tested by ICA for OXA 23. The ICA kit revealed the negative result from all non-A. baumannii (Table $3 \& 4$ ).

\section{DISCUSSION}

Although rapid detection methods such as real-time PCR assay are used routinely to control the spread of the infection by multidrug-resistant (MDR) microorganism, including CRAB, MRSA, VRE in many clinical laboratories, conventional bacterial cultures are used to isolate MDR microorganism from clinical specimens and to confirm the presence of MDR in specimens containing a mixed bacterial population. Moreover, this approach allows antimicrobial susceptibility testing for selection of an appropriate anti-
Table 3. Results of immunochromatographic assay for OXA-23 $\beta$-lactamase in gram positive cocci and yeasts

\begin{tabular}{lcc}
\hline \hline \multicolumn{1}{c}{ Species } & A-Lab & B-Lab \\
\hline Staphylococcus aureus ATCC 29213 & Negative & Negative \\
Staphylococcus aureus & Negative & Negative \\
Streptococcus pneumoniae & Negative & Negative \\
Streptococcus agalactiae & Negative & Negative \\
Streptococcus viridans group & Negative & Negative \\
Enterococcus faecalis ATCC 29212 & Negative & Negative \\
Enterococcus faecalis & Negative & Negative \\
Enterococcus faecium & Negative & Negative \\
Staphylococcus epidermidis & Negative & Negative \\
Staphylococcus hominis & Negative & Negative \\
Candida albicans & Negative & Negative \\
Candida parapsilosis & Negative & Negative \\
Candida trophicalis & Negative & Negative \\
\hline
\end{tabular}

microbial treatment. After CRAB have been cultured and identified, molecular methods are necessary to determine their genotype (Class B or D: OXA genotypes); such methods are labor-intensive and costly yet indispensable, and their use other than in epidemiological investigations is controversial. 
Table 4. Results of immunochromatographic assay for OXA-23 $\beta$-lactamase in gram negative bacilli

\begin{tabular}{lcc}
\hline \hline \multicolumn{1}{c}{ Species } & A-Lab & B-Lab \\
\hline Escherichia coli ATCC 25922 & Negative & Negative \\
Pseudomonas aeruginosa & Negative & Negative \\
ATCC 27853 & Negative & Negative \\
Klebsiella oxytoca ATCC 700324 & Negative & Negative \\
Escherichia coli & Negative & Negative \\
Klebsiella pneumoniae & Negative & Negative \\
Klebsiella oxytoca & Negative & Negative \\
Enterobacter aerogenes & Negative & Negative \\
Enterobacter cloacae & Negative & Negative \\
Pseudomonas aeruginosa & Negative & Negative \\
Stenotrophomonas maltophilia & Negative & Negative \\
Burkholderia cepacia & Negative & Negative \\
Acinetobacter baumannii (IMP-S) & Negative & Negative \\
Pseudomonas putida & &
\end{tabular}

Abbreviation; IMP-S, imipenem susceptible

The immunological assay may be useful for confirming the genotype of CRAB in this situation, which is encountered daily in microbiological laboratories worldwide. Moreover, this OXA-type carbapenemase ICA can incorporate multiple test lines and will facilitate control of nosocomial infection by OXA-23 CRAB together with ICAs for various OXA-type carbapenemase. In this study, we developed a specific mAbs for rapid detection of OXA-23 $\beta$-lactamase. Seven mAbs to OXA-23 $\beta$-lactamase were selected, one pair (6G4/4G6) mAb showed the greatest reactivity. However, the ICA using this $\mathrm{mAb}$ showed a weak signal in the kit. To improve the sensitivity of ICA kit, a rabbit polyclonal antibody by recombinant OXA-23 $\beta$-lactamase was developed and was used as a capture antibody of ICA with detector antibody (6G4 mAb). The kit produced a distinct signal and the rabbit polyclonal antibody was used capture antibody of ICA (Fig. 2).

Of 102 A. baumannii isolates, 61 isolates were positive for $b l a_{\mathrm{OXA}-23}$ gene and 41 isolates were negative for $b l a_{\mathrm{OXA}-23}$ gene by PCR analysis. Sixty isolates among 61 isolates with $b l a_{\text {OXA-23 }}$ gene were positive by ICA. Forty isolates among 41 isolates without $b l a_{\text {OXA-23 }}$ gene were negative by ICA in A-laboratory (Table 2). In addition, except for two false negative results, 100 of 102 isolates were consistent with PCR results in B-laboratory. The overall sensitivity and specificity were $98.36 \%$ and $97.56 \%$ in A-laboratory, $96.72 \%$ and $100 \%$ in B-laboratory, respectively (Table 2). To evaluate cross reactivity with other bacteria, the carbapenem resistant $P$. aeruginosa, $A$. xylosoxidans and the other gram negative or positive bacteria were tested by ICA for OXA-23. The ICA kit revealed a negative result from all non-A. baumannii (Table 3 ).

The limitation of this study is that the performance of the developed ICA kit was evaluated using the colony grown on agar plates rather than direct clinical samples for overcoming the low sensitivity of this kit. Therefore, the newly developed ICA kit requires the more consuming time than a PCR method to directly detect OXA-23 $\beta$-lactamase from clinical samples. In addition, a considerable quantity of bacterial colony is required to test of ICA kit. Furthermore, the evaluation for detection limit and conditions to improve the sensitivity, such as enrichment incubation with carbapenem, which induce the OXA-23 $\beta$-lactamase, are required.

However, because rapid and easy detection of CRAB is the primary object of ICA and most laboratory usually isolated bacteria on agar plates, ICA is sufficient to detect OXA-23 producing $A$. baumanii in clinical laboratories. The cost of using this kit is estimated to be only $10 \%$ of the cost of performing real-time PCR, which is the current method of detecting the OXA-23 $\beta$-lactamase gene in South Korea, making it affordable for most clinical laboratories. In addition, whereas many molecular methods require batch operations by trained personnel, this rapid kit assay can be used with few samples and by non-specialist workers (Shin et al., 2010; Ji et al., 2014).

In conclusion, the novel ICA assay is easy-to-use, and rapid immunologic method for the detection of OXA-23 $\beta$-lactamase-producing A. baumannii from conventionally cultured bacterial colonies. To our knowledge, this is the first report of an immunological detection assay for OXA-23 $\beta$-lactamase. Additional development of rapid immunological assays for OXA-24, OXA-48 and OXA-51 will facilitate the control of $\mathrm{CRAB}$ infection and investigation of its epidemiology. 


\section{Acknowledgements}

This work was supported by the Research Grant of Small and Medium Business administration of Korea (Project no. S2082829).

\section{Conflict of interest}

The authors have no conflicts of interest to disclose.

\section{REFERENCES}

Bergmans HEN, van Die IM, Hoekstra WPM. Transformation in Escherichia coli: stages in the process. J Bacteriol. 1981. 146: 564-570.

Clinical and Laboratory Standard Institute. Performance for antimicrobial susceptibility testing; twenty two informational supplement (M100-S22) Wayne, PA:CLSI, 2012.

Hochuli E, Bannwarth W, Döbeli H, Gentz R, Stüber D. Genetic approach to facilitate purification of recombinant proteins with a novel metal chelate adsorbent. Nature Biotechnology. 1988. 6: 1321-1325.

Jeong SH, Bae IK, Park KO, An YJ, Sohn SG, Jang SJ, Sung KH, Yang KS, Lee K. Outbreaks of imipenem-resistant Acinetobacter baumannii producing carbapenemases in Korea. J Microbiol. 2006. 44: 423-431.

Ji GY, Song HG, Son BR, Hong SB, Kim JW, Shin KS. Development of a novel immunochromatographic assay for rapid detection of VanA ligase-producing vancomycin-resistant enterococci. J Microbiol Biotechnol. 2014. 24: 427-430.

Lee H, Yong D, Yum JH, Roh KH, Lee K, Yamane K, Arakawa Y, Chong Y. Dissemination of 16S rRNA methylase-mediated highly amikacin-resistant isolates of Klebsiella pneumoniae and Acinetobacter baumannii in Korea. Diagn Microbiol Infect Dis. 2006. 56: 305-312.

Lee K, Lim YS, Yong D, Yum JH, Chong Y. Evaluation of the Hodge test and the imipenem-EDTA double-disk synergy test for differentiating metallo-beta-lactamase-producing isolates of Pseudomonas spp. and Acinetobacter spp. J Clin Microbiol. 2003. 41: 4623-4629.

Lee JH, Kang HY, Lee JY, Kim J, Lee YC, Seol SY, Cho DT, Kim KW, Song DY, Lee JC. Differences in phenotypic and genotypic traits against antimicrobial agents between Acinetobacter baumannii and Acinetobacter genomic species 13TU. J Antimicrob Chemother. 2007. 59: 633-639.

Lee K, Kim MN, Choi TY, Cho SE, Lee S, Whang DH, Yong D,
Chong Y, Woodford N. Wide dissemination of OXA-type carbapenemases in clinical Acinetobacter spp. isolates from South Korea. Int J Antimicrob Agents. 2009. 33: 520-524.

Lee K, Kim MN, Kim JS, Hong HL, Kang JO, Shin JH, Park YJ, Yong D, Jeong SH. Further increases in carbapenem-, amikacin-, and fluoroquinolone-resistant isolates of Acinetobacter spp. and P. aeruginosa in Korea: KONSAR study 2009. Yonsei Med J. 2009. 52: 793-802.

Munoz-Price LS, Weinstein RA. Acinetobacter infection. N Engl J Med. 2008. 358: 1271-1281.

Peleg AY, Seifert H, Paterson DL. Acinetobacter baumannii: emergence of a successful pathogen. Clin Microbiol Rev. 2008. 21: 538-582.

Shin KS, Song HG, Kim H, Yoon S, Hong SB, Koo SH, Kim J, Kim JW, Rho KH. Direct detection of methicillin-resistant Staphylococcus aureus from blood cultures using a MRSA rapid kit based on immunochromatographic immunoassay to detect penicillin-binding protein 2a. Diagn Microbiol Infect Dis. 2010. 67: 301-303.

Stähli C, Staehelin T, Miggiano V, Schmidt J, Häring P. High frequencies of antigen-specific hybridoma: dependence on immunization parameters and prediction by spleen cell analysis. J Immunnol Methods. 1980. 32: 297-304.

Sung JY, Kwon KC, Cho HH, Koo SH. Antimicrobial resistance determinants in imipenem-nonsusceptible Acinetobacter calcoaceticus-baumannii complex isolated in Daejeon, Korea. Korean J Lab Med. 2011. 31: 265-270.

Turton JF, Woodford N, Glover J, Yarde S, Kaufmann ME, Pitt TL. Identification of Acinetobacter baumannii by detection of the blaOXA-51-like carbapenemase gene intrinsic to this species. J Clin Microbiol. 2006. 44: 2974-2976.

Villegas MV, Hartstein AI. Acinetobacter outbreaks, 1977-2000. Infect Control Hosp Epidemiol. 2003. 24: 284-295.

Walther-Rasmussen J, Hoiby N. OXA-type carbapenemases. J Antimicrob Chemother. 2006. 57: 373-383.

Wilks M, Wilson A, Warwick S, Price E, Kennedy D, Ely A, Millar MR. Control of an outbreak of multidrug-resistant Acinetobacter baumannii-calcoaceticus colonization and infection in an intensive care unit (ICU) without closing the ICU or placing patients in isolation. Infect Control Hosp Epidemiol. 2006. 27: 654-658.

Woodford N, Ellington MJ, Coelho JM, Turton JF, Ward ME, Brown S, Amyes SG, Livermore DM. Multiplex PCR for genes encoding prevalent OXA carbapenemases in Acinetobacter spp. Int J Antimicrob Agents. 2006. 27: 351-353. 
Yum JH, Yong D, Lee K, Kim HS, Chong Y. A new integrin carrying VIM-2 metallo-b-lactamase gene cassette in a Serratia marcescnes isolate. Diagn Microbiol Infect Dis. 2002. 42: 217 -219 .
Zong Z, Lu X, Valenzuela, JK, Partridge SR, Iredell J. An outbreak of carbapenem-resistant Acinetobacter baumannii producing OXA-23 carbapenemase in western China. Int J Antimicrob Agents. 2008. 31: 50-54. 